BMJ Surgery, Interventions, \& Health Technologies

\section{Quantifying asymmetry of anterior cerebral arteries as a predictor of anterior communicating artery complex aneurysm}

To cite: Burlakoti A, Kumaratilake J, Taylor DJ, et al. Quantifying asymmetry of anterior cerebral arteries as a predictor of anterior communicating artery complex aneurysm. BMJ Surg Interv Health Technologies 2020;2:e00059. doi:10.1136/ bmjsit-2020-000059

- Additional material is published online only. To view please visit the journal online (http://dx.doi.org/10.1136/ bmjsit-2020-000059).

Received 25 July 2020 Revised 29 September 2020 Accepted 16 November 2020

Check for updates

(C) Author(s) (or their employer(s)) 2020. Re-use permitted under CC BY-NC. No commercial re-use. See rights and permissions. Published by BMJ.

${ }^{1}$ UniSA Allied Health and Human Performance, University of South Australia, Adelaide, South Australia, Australia

${ }^{2}$ Discipline of Anatomy and Pathology, Adelaide Medical School, The University of Adelaide Faculty of Health Sciences, Adelaide, South Australia, Australia

${ }^{3}$ SA Medical Imaging, Royal Adelaide Hospital, Adelaide, South Australia, Australia ${ }^{4}$ Institute of Evolutionary Medicine, University of Zurich Faculty of Medicine, Zurich, ZH, Switzerland

Correspondence to

Dr Arjun Burlakoti;

Arjun.Burlakoti@unisa.edu.au

\section{ABSTRACT}

Objectives The aim of this study was to establish an anatomical index for early prediction of the risk of development of aneurysms in anterior communicating arterial complex (AcomAC). The asymmetric diameter of one anterior cerebral artery (ACA) to other could alter haemodynamics and may contribute to formation of aneurysms in AcomAC and be a reliable predictor of the risk of development of aneurysms.

Design and setting This is a retrospective, observational and quantitative study, which used cerebral computed tomography angiography (CCTA) scans in South Australia. Participants CCTA scans of 166 adult patients of both sexes were studied.

Main outcome measures The internal diameters of the proximal segments of ACAs (A1s) were measured. Position and presence or absence of aneurysms in AcomAC were determined. The ratio of $\mathrm{A} 1$ diameters was taken as a measure of $A 1$ asymmetry.

Results The ratio of diameters of $A 1 s$ correlated with the occurrence of AcomAC aneurysms. The risk of development of aneurysms in AcomAC was much greater $(80 \%, 0 R=47.3)$ when one $A 1$ segment's radius was at least 50\% larger (ie, 2.25 times cross-sectional area) than the other.

Conclusion The general information on asymmetric A1 has been published previously. The present findings have significant contribution since the A1s asymmetry ratios have been categorised in ascending order and matched with the presence of AcomAC aneurysms. The asymmetry ratio of the $A 1$ is a good predictor for the development of AcomAC aneurysms. Reconstruction of the asymmetric A1 could be done if the technology gets advanced.

\section{INTRODUCTION}

Rupture of cerebral aneurysms causes subarachnoid haemorrhages (SAH) leading to high mortality and morbidity. The incidence of SAH has been 10-36 per 100000 people per year and about $3 / 4$ of them resulted from spontaneous rupture of cerebral aneurysms. ${ }^{12}$ Large cerebral aneurysms may also compress adjacent cranial nerves. ${ }^{3}$ The mortality and morbidity rates resulting from ruptured cerebral aneurysms remain
Key messages

What is already known about this subject?

- Relationship of asymmetry of A1 segment of anterior cerebral artery (ACA) to the occurrence of aneurysms in anterior communicating arterial complex (AcomAC) has been observed in literature but has not been explained nor quantified.

What are the new findings?

- Asymmetry of the A1 of ACA was quantified, and a mathematical model has been established to predict the likelihood of developing AcomAC aneurysms depending on the degree of asymmetry.

How might these results affect future research or surgical practice?

- Patients with asymmetry of $A 1$ found in their brain scans should be closely followed up, because of the high risk of developing aneurysms in the AcomAC complex. Reconstruction of the asymmetric A1 can be done to prevent the development of AcomAC aneurysms, if ethically justified.

high, with around one-third dying at the time, one-third suffering a major stroke and one-third making a reasonable recovery. ${ }^{4}$ An aneurysm is a dilatation and outpouching of the wall of a blood vessel. ${ }^{56}$ The action of fluctuating blood pressure on vascular walls has been identified as the main cause for the development of aneurysms. ${ }^{7}$ The risk of aneurysm rupture is 6-8 in 100000 per year in most developed countries. ${ }^{8}$ In South Australia, where the total population is 1.7 millions, radiologists involved in the treatment observed approximately 170 ruptured aneurysm cases per year (ie, 1 in every 10000 cases per year). Another study revealed that about 1 in 30 adults likely to have intracranial aneurysms and in 25\% of them, the aneurysms could rupture and produce SAHs or compression of surrounding structures. ${ }^{7}$ Anterior communicating artery complex (AcomAC) has been the most common 
location of ruptured cerebral aneurysms. ${ }^{9}$ Unruptured aneurysms have been observed in $2.8 \%$ of patients investigated by magnetic resonance (MR) angiography. ${ }^{10}$ People with variations in cerebral arteries, particularly, in anterior cerebral arterial (ACA) territory are thought to be subjected to imbalance in cerebral blood flow leading to cerebrovascular pathologies, including cerebral aneurysms. ${ }^{11}$

Variations in cerebral basal arterial network have ranged from missing arterial segments to asymmetry between collateral arterial segments and the later was more common. ${ }^{12}{ }^{13}$ Some of the most variant and asymmetric patterns of arteries were seen in relation to anterior cerebral and anterior communicating arterial territories. ${ }^{11} 14$ Fluctuation in arterial blood flow, and thus the blood pressure, has been observed in asymmetric A1 segments. ${ }^{15} 16$ Such variations in blood flow could predispose the arterial wall for aneurysmal dilatations. ${ }^{17-22}$ Genetics, smoking, trauma and medications are factors that could weaken the walls of arteries and predispose them to the development of aneurysms, particularly when subjected to alteration in haemodynamics or chronic hypertension. ${ }^{22}$ Prediction and early detection of aneurysms allow treatment, thus could prevent or reduce the incidences of cerebral stroke, including reoccurrences of aneurysms. The aim of this research was to investigate the relationship of asymmetry between A1s and the development of AcomAC aneurysms. As far as we know, no studies have been done on quantifying and calculating the degree of A1 asymmetry to the occurrences of AcomAC aneurysms. A method to predict the risk of occurrence of aneurysms in AcomAC using the degree of asymmetry between right and left A1s has been established.

\section{METHODS}

\section{Study design}

Internal diameters of A1s were measured on cerebral CT angiography (CCTA) digital images obtained from 166 (80 males and 86 females) adult individuals (average age $=60$ years, $\mathrm{SD}=16$ ) (see online supplemental file 1 ). The same images were used to determine the presence or the absence of AcomAC aneurysms. The source of the CCTA images were the Carestream (Vue RIS V.11.0.14.35) database of the Royal Adelaide Hospital (RAH), University of Adelaide, South Australia. The CCTA images were taken between January 2011 and December 2018. Patient's personal details have not been copied, documented or included in this research.

The CCTA images studied were those taken for the clinical investigation of different cranial pathologies. These included 51 cases out of 166 patients who had a history of previously diagnosed cerebral aneurysms (see online supplemental file 1 ).

\section{Data collection and extraction}

Data collection was carried out by the corresponding author in consultation with radiologists from the RAH,

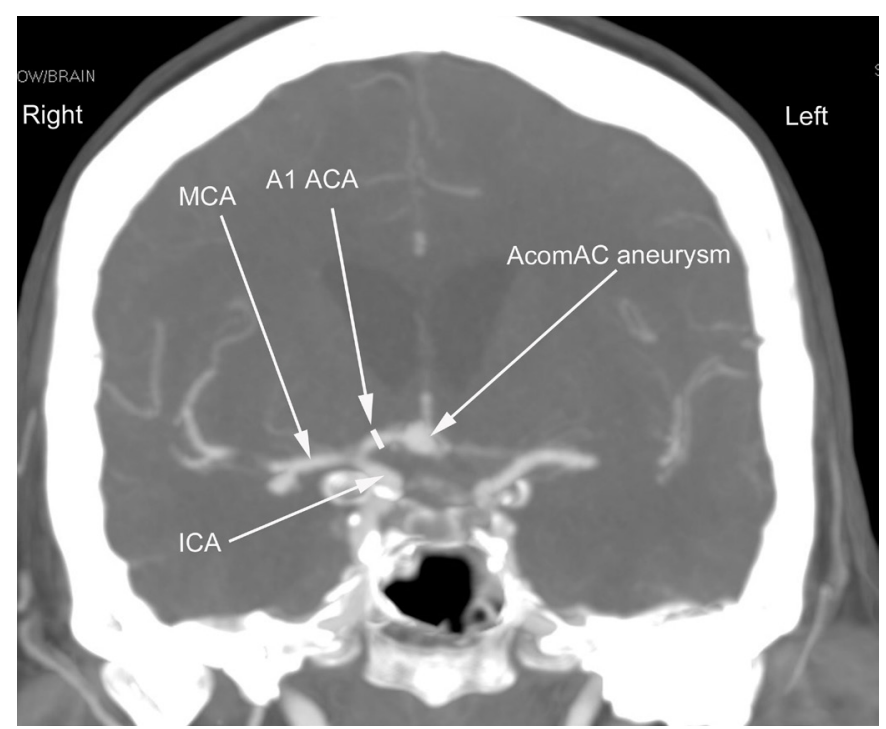

Figure 1 Cerebral CT Angiography scan with AcomAC aneurysm in coronal view, white line perpendicular to the long axis of the vessel (A1 ACA) indicates measurement site. A1 ACA, the first part of anterior cerebral artery; AcomAC=anterior communicating arterial complex; ICA, internal carotid artery; MCA, middle cerebral artery.

South Australia who were involved in patients' care. Cases with severe cerebral vasospasm diagnosed by radiologists and recorded in the data system were excluded from the study. The internal diameters were measured at the midpoint of the left and right A1s were measured perpendicular to the long axis of the vessels at the narrowest possible sites (in the coronal and axial CCTA images) using image J software (figure 1, online supplemental figure 1 and online supplemental figure 2). Measurements taken by the image $\mathrm{J}$ software have been proven accurate and reliable in previous studies. ${ }^{23}$ The reliability and the accuracy of the measurements were confirmed by repeating measurements of 30 cases at 15 months interval by the same person and determined the intra-observer errors (table 1 and online supplemental file 2). Comparison of first and repeated measurements gave a $10 \%$ relative technical error of measurement (rTEM) without adjustment and less than 5\% rTEM with minor correction and adjustment (table 1 and online supplemental file 2). These reliability and the accuracy calculations were statistically acceptable. ${ }^{24}$

The selection of measurement sites was consistent throughout the data collection. Data were taken only from the electronic files stored in the Carestream software at the RAH, University of Adelaide. Occurrence of AcomAC aneurysms with or without the presence of aneurysm elsewhere and prior history of aneurysms anywhere in the brain were included from each individual case.

The asymmetry ratios of right and left or left and right (ie, bigger to smaller ratio) A1 arteries were computed for all 166 CCTA cases (see online supplemental file 1). The calculated bigger to smaller A1 asymmetry ratios were categorised into three groups (ie, mild to moderate asymmetry $\leq 1.5$, substantial asymmetry $>1.5$ to $\leq 2$ and 
Table 1 Accuracy and reliability of the measurements of the first part of the anterior cerebral artery (A1) in cerebral CT angiography (CTA) scans

\begin{tabular}{|c|c|c|c|}
\hline & Reliability (R) & $\begin{array}{l}\text { Technical error of } \\
\text { measurement (TEM) } \\
\text { in } \mathrm{mm}\end{array}$ & $\begin{array}{l}\text { Relative TEM (rTEM) } \\
\text { in } \%\end{array}$ \\
\hline Repeat A1 measurement in axial CTA images (not adjusted*) & 0.93 & 0.24 & 11.00 \\
\hline Repeat A1 measurement in axial CTA images (adjusted) & 0.98 & 0.12 & 5.39 \\
\hline Repeat A1 measurement in axial and coronal CTA images (adjusted ${ }^{\star}$ ) & 0.98 & 0.10 & 4.77 \\
\hline Repeat A1 measurement in coronal CTA images (not adjusted) & 0.94 & 0.22 & 10.45 \\
\hline
\end{tabular}

The coefficients of variation of the measurements ( $\mathrm{rTEM}$ ) are presented. Reliability is the correlation among the previous first measurement done in coronal and axial CTA slices and the second measurement performed in axial and coronal cerebral CTA after 15 months of the initial measurement taken of the same artery, computed tomography $=C T, A 1=$ first part of anterior cerebral artery, $n=30$ *Adjustment made by excluding two outliers from the previous and the corresponding repeat measurements of right A1 (file supplied in online supplemental file 2).

severe asymmetry $\geq 2$, (table 2 and online supplemental file 3). The rationale for this classification was for easy application and interpretation. The diameter (and hence also the radius) ratio of 1.5 corresponds to the 2.25 times difference in the cross-sectional area of the vessel's lumen, while the ratio of 2.0 reflects four times difference in the cross-sectional area of the vessel lumen.

\section{STATISTICAL ANALYSIS}

A cross-sectional observational design and SPSS V.25 (IBM) program were used in the study. Measurement error analysis has been described in table 1 . In the main analysis, non-parametric statistics $\left(\chi^{2}\right)$ were used and ORs were calculated to observe the strength of association between the A1 asymmetries and the AcomAC aneurysms (table 2 and online supplemental file 3 ).

\section{RESULTS}

The asymmetry ratios of right and left A1 segments of ACA together with the presence and absence of AcomAC aneurysms are presented in table 2, and online supplemental files 1 and 3 (in ascending order, $\mathrm{n}=166$ ). Among 141 patients with mild to moderate A1 asymmetry $(\leq 1.5)$, 11 had AcomAC aneurysms. Out of 13 patients with substantial asymmetry ( $>1.5$ to $\leq 2.0), 10$ had AcomAC aneurysms. In 12 patients with severe type of asymmetric ratios (>2.0), 10 were affected with AcomAC aneurysms

Table 2 Probability (out of 1.00) to have anterior communicating artery complex aneurysms in relation to the degree of right and left asymmetricity of A1 ACA

\begin{tabular}{|c|c|c|c|c|c|c|}
\hline \multirow[t]{2}{*}{$\begin{array}{l}\text { A1 asymmetry ratio (right and left bigger to smaller } \\
\text { A1) }\end{array}$} & \multicolumn{2}{|c|}{$\begin{array}{r}\text { AcomA } \\
\text { complex } \\
\text { aneurysm } \\
\end{array}$} & \multirow[b]{2}{*}{ Total } & \multirow[b]{2}{*}{$\%$ Chance } & \multirow[b]{2}{*}{ OR } & \multirow{2}{*}{$\begin{array}{r}\chi^{2} \text { (asymptotic } \\
\text { significance); } p \\
\text { value }\end{array}$} \\
\hline & No & Yes & & & & \\
\hline \multicolumn{7}{|l|}{ No of cases; $n=166$} \\
\hline Mild to moderate $(\leq 1.5)$ & 130 & 11 & 141 & 7.8 & 0.02 & 0.0001 \\
\hline Substantial to severe $(>1.5)$ & 5 & 20 & 25 & 80.0 & 47.3 & 0.0001 \\
\hline Total & 135 & 31 & 166 & & & \\
\hline \multicolumn{7}{|l|}{ No of cases without history of aneurysm $(n=115)$} \\
\hline Mild to moderate $(\leq 1.5)$ & 93 & 7 & 100 & 7.0 & 0.027 & 0.0001 \\
\hline Substantial to severe $(>1.5)$ & 4 & 11 & 15 & 73.3 & 36.53 & 0.0001 \\
\hline Total & 97 & 18 & 115 & & & \\
\hline \multicolumn{7}{|l|}{ No of cases with history of aneurysm $(n=51)$} \\
\hline Mild to moderate $(\leq 1.5)$ & 37 & 4 & 41 & 9.6 & 0.012 & 0.0001 \\
\hline Substantial to severe $(>1.5)$ & 1 & 9 & 10 & 90.0 & 83.25 & 0.0001 \\
\hline Total & 38 & 13 & 51 & & & \\
\hline
\end{tabular}

$n=166$, without history of aneurysms $(n=115)$, with the previous history of aneurysm $(n=51)$.

A1 ACA, first segment of the anterior cerebral artery; AcomA, anterior communicating artery. 
Table 3 Presence or absence of anterior communicating artery complex (AcomAC) aneurysms and cerebral aneurysms elsewhere in the current study

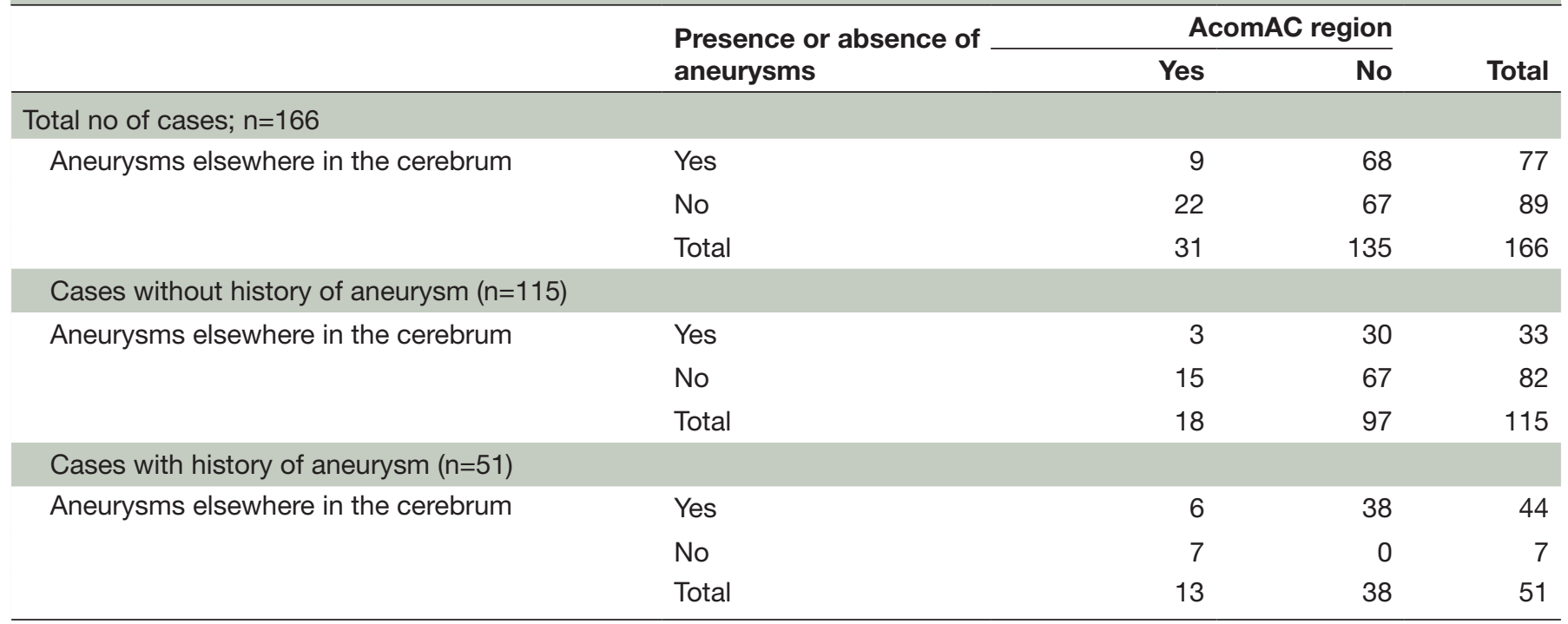

$\mathrm{n}=166$; without history of aneurysms, $\mathrm{n}=115$; and with the previous history of aneurysm, $\mathrm{n}=51$.

(table 2, online supplemental file 3). Among the people with A1 asymmetry ratios of less than 1.5 just $7.8 \%$ had aneurysms while in those with ratios of $>1.5$ to $\leq 2$ and $>2$, the risks of developing AcomAC aneurysm were $77 \%$ and $83 \%$, respectively. In summary, patients with asymmetry ratios greater than 1.5 had $80 \%$ risk of developing aneurysms $(\mathrm{OR}=47.3)$, while those with asymmetry ratios below 1.5 had $7.8 \%$ AcomAC aneurysms $(\mathrm{OR}=0.02)$, (table 2, online supplemental files 1, 3 and 4).

The chances of developing AcomAC aneurysms in the presence of substantial and severe A1 asymmetries were statistically similar between people with or without a previous history of aneurysms (table 2 and online supplemental file 3). In patients with no previous history of aneurysms $(n=115)$, the incidences, risks and ORs (table 2 and online supplemental file 3) of AcomAC aneurysms were similar to the entire sample and to the patients with previous history of aneurysm.

The prevalence of AcomAC aneurysms between sexes and among age groups was statistically not different. Altogether, 31 out of 166 cases had AcomAC aneurysms and 77 out of 166 cases had cerebral aneurysms elsewhere (ie, other than the AcomAC aneurysm). Seven out of 11 cases with AcomAC aneurysms that had only mild asymmetry of A1 also had aneurysms elsewhere in the brain other than AcomAC location (table 3). However, there was no significant relationship between the presence of AcomAC aneurysms and aneurysms elsewhere in the brain $\left(\chi^{2} 3.7\right.$, $\mathrm{p}=0.05)$. Furthermore, statistical relationship between A1 asymmetricity and the presence of cerebral aneurysms elsewhere was not found $(\mathrm{p}>0.05)$. All patients with AcomAC aneurysms had 1.66 median asymmetry ratio, while the patients without the presence of AcomAC aneurysms had 1.09 median asymmetry ratio. The median A1 asymmetry ratio for all the cases included in this study was just 1.10 (table 3, online supplemental files 1 and 3).

\section{DISCUSSION}

The current study quantified for the first time, A1 asymmetry and the likelihood of occurrences of AcomAC aneurysms. Previously the co-occurrence of AcomAC aneurysms with A1 asymmetry has been observed but not quantified. $^{112526}$ The study included random CCTA cases accessing the data at a specialised tertiary centre. Obviously, we would assume to see cases of suspected cerebral pathologies in a specialised tertiary medical centre. We examined fairly a large number of 166 CTA evaluating individual A1 asymmetry and aneurysms. The findings (OR and risk percentage) on A1 asymmetry ratio $(\geq 1.42)$ were extremely significant in relation to the AcomAC aneurysms.

The findings of the study indicate that, the prevalence of aneurysms in AcomAC was greater with increasing asymmetry between left and right A1s (table 2 and online supplemental file 3 ). The asymmetry ratio of 1.5 indicates that the cross-sectional area of an A1 segment is twice as large as that of the other one $\left(1.5^{2}=2.25\right)$. Furthermore, such asymmetry would likely to have significant haemodynamic effects that could produce $80 \%$ risk of AcomAC aneurysms (table 2, online supplemental file 3 and online supplemental file 4). The exact mechanism involved in causing aneurysms in AcomAC is not well understood. ${ }^{27}$ The development of aneurysm could be due to the altered haemodynamics resulting from the increased blood flow and the greater peak systolic pressure in the larger ACA. ${ }^{12} 2728$ Imbalanced haemodynamics originating from the larger ACA may weaken and dilate the wall of the AcomAC at branching points, resulting in an 
aneurysmal formation. ${ }^{12} 2129$ Thus, the extent of the asymmetry in A1s may allow to predict the occurrence of the AcomAC aneurysms. Current sample included patients presenting with various cerebral problems, including strokes and aneurysms. However, when patients were divided into two subsamples: those with a history and without known history of aneurysms, the results did not differ significantly between these sub samples (table 2 and online supplemental file 3 ). This lack of difference indicates that prior history of aneurysms did not influence the overall results of the study. Therefore, the observed correlation between asymmetry of A1s and AcomAC aneurysms is independent of the prior history of any cerebral aneurysms, because in the current sample there is no correlation between presence of AcomAC aneurysms and aneurysms elsewhere ((table 2 and online supplemental file 3).

The A1 asymmetry ratio was just below 1.5 in 11 out of 31 AcomAC aneurysms cases. However, 3 out of those 11 cases had A1 asymmetry ratios of more than 1.42 (indicating double the cross-sectional area of one A1 artery compared with the other). Furthermore, all others (ie, 8 out of those 11 cases with asymmetry ratio below 1.5) had asymmetry ratios above the median of 1.09 and represented the 'mild to moderate asymmetry' category. Seven of those 11 cases had also aneurysms elsewhere (tables 2 and 3 and online supplemental file 3). These may indicate that causes for the development of AcomAC aneurysms in the lower A1 asymmetry $(<1.40)$ cases may be because of the quality of vessel's walls and high blood pressure, in addition to altered haemodynamics resulting from the asymmetry.

Since the CCTA data were taken from the specialised medical centre, it is true that we get to see symptomatic individuals with different pathologies. That approach is even better to see the connection between A1 asymmetry and the presence or absent of aneurysms rather than trying to scan many innocent people in the community, exposing them to the radiation unnecessarily. Modifiable known risk factors, such as history of smoking and hypertension were not quantified in this study. These could have been supplementary factors promoting AcomAC aneurysms, however, literature suggests hypertension is not related to the cerebral aneurysms. ${ }^{30}$ Furthermore, there is no reason to assume that A1 asymmetry is related to the smoking and hypertension. This research found a coincidence of A1 asymmetry and AcomAC aneurysms. This coincidence could result from: (A) AcomAC aneurysm altering the blood flow and remodelling the size of A1 segments, (B) Asymmetry of A1 arteries causing altered blood flow in AcomAC and affecting the walls and producing the aneurysm. Remodelling of the size of arteries in the vicinity or proximal to an aneurysm is not known, therefore, it is more likely that $\mathrm{A} 1$ asymmetry causes aneurysms. A longitudinal prospective study would likely confirm vessel asymmetry as the cause of aneurysms rather than the reverse. We are not aware of such a study being conducted and there may be significant ethical impediments. Treatment and the management of patients after strokes are costly to the affected family as well as to the country. A multinational study has shown that, the cost of management of a patient after a stroke ranged from US $\$ 18538$-US $\$ 228038 .{ }^{31}$ The procedure of treatment of unruptured aneurysms is safe, and the risk of development of stroke is approximately $3 \%$ and the mortality is less than $1 \%$, therefore, there is great advantage in identifying and treating aneurysms before they rupture. ${ }^{32} 33$

The ability to predict the likelihood of the development of aneurysms in AcomAC using the asymmetry ratio between right and left A1s could enhance the viability of a national screening programme.

Undertaking CCTA screening in the general population is not recommended due to ethical reason. However, if A1 asymmetry is noticed in cranial investigation done for other reasons, clinicians should be cautious as it could indicate the possibility of future development of aneurysms. Therefore, MRI screening of older individuals may be beneficial, and has been recommended. ${ }^{20}$ These findings make significant contribution to existing knowledge, since the A1 asymmetry index has been categorised in ascending order and matched with the presence or absence of AcomAC aneurysms in each of the 166 cases. This type of study has not been done before. General anatomical variations of $\mathrm{A} 1$ could be corrected with the advancement of medical and surgical technologies. This would prevent unequal blood flow and pressure contributing to the occurrences of AcomAC aneurysms. Patients who have A1 asymmetry (especially the A1 asymmetry with $\geq 1.42$ ) on scans should be monitored regularly by follow up imaging and angiograms. Reconstruction of A1 asymmetry is a future possibility with technological advancement.

\section{CONCLUSION}

The asymmetry of the diameters of A1s should be routinely assessed in all patients undergoing cerebral imaging, which includes these vessels. Patients with asymmetry of the A1 should be closely followed up, because of the high risk of development of aneurysms in the AcomAC complex. Reconstruction of the asymmetric A1 could be done if the technology gets advanced in the future.

Contributors $A B$ conceived the idea, designed the analysis, collected and analysed the data from cerebral CT angiography (CCTA), took pictures, recorded videos, contributed in conceptualisation, prepared and drafted the manuscript. JK conceived the idea, contributed to the concept, helped in data interpretation, editing and the critical revision of the manuscript and approving the article. DJT conceived the idea, contributed in collecting and interpreting the data, editing the manuscript, the critical revision of the manuscript and approving the article. MH conceived the idea, helped in statistics, data analysis and interpretation, editing the manuscript, the critical revision of the manuscript and in approving the article.

Funding The authors have not declared a specific grant for this research from any funding agency in the public, commercial or not-for-profit sectors.

Competing interests None declared.

Patient consent for publication Not required. 
Ethics approval The use of these information for research was approved by the University of Adelaide Human Research Ethics Board (approval number: H2014176).

Provenance and peer review Not commissioned; externally peer reviewed.

Data availability statement Data are available on reasonable request. All data relevant to the study are included in the article or uploaded as online supplemental information. Data are available on request, please feel free to email Arjun.

Burlakoti@unisa.edu.au

Open access This is an open access article distributed in accordance with the Creative Commons Attribution Non Commercial (CC BY-NC 4.0) license, which permits others to distribute, remix, adapt, build upon this work non-commercially, and license their derivative works on different terms, provided the original work is properly cited, appropriate credit is given, any changes made indicated, and the use is non-commercial. See: http://creativecommons.org/licenses/by-nc/4.0/.

\section{ORCID iD}

Arjun Burlakoti http://orcid.org/0000-0001-9317-6352

\section{REFERENCES}

1 Nilsson OG, Lindgren A, Ståhl N, et al. Incidence of intracerebral and subarachnoid haemorrhage in southern Sweden. J Neurol Neurosurg Psychiatry 2000;69:601-7.

2 Mitchell P, Jakubowski J. Estimate of the maximum time interval between formation of cerebral aneurysm and rupture. J Neurol Neurosurg Psychiatry 2000;69:760-7.

3 Yanaka K, Matsumaru Y, Mashiko R, et al. Small unruptured cerebral aneurysms presenting with oculomotor nerve palsy. Neurosurgery 2003;52:553-7.

4 Sekhar LN, Morton R. Risk factors for three phases of 12-month mortality in a defined population after subarachnoid hemorrhage. World Neurosurg 2012;78:579-80.

5 Fisher CM. Cerebral miliary aneurysms in hypertension. Am J Pathol 1972;66:313.

6 Forbus WD. On the origin of miliary aneurysms of superficial cerebral arteries. Bulletin of The Johns Hopkins Hospital 1930;47:239-48.

7 Korja M, Kaprio J. Controversies in epidemiology of intracranial aneurysms and SAH. Nat Rev Neurol 2016;12:50-5

8 Zacharia BE, Hickman ZL, Grobelny BT, et al. Epidemiology of aneurysmal subarachnoid hemorrhage. Neurosurg Clin N Am 2010;21:221-33.

9 Ye J, Zheng P, Hassan M, et al. Relationship of the angle between the $A 1$ and $A 2$ segments of the anterior cerebral artery with formation and rupture of anterior communicating artery aneurysm. J Neurol Sci 2017;375:170-4.

10 Horikoshi T, Akiyama I, Yamagata Z, et al. Retrospective analysis of the prevalence of asymptomatic cerebral aneurysm in 4518 patients undergoing magnetic resonance angiography--when does cerebral aneurysm develop? Neurol Med Chir 2002;42:105-13.

11 Brust JCM, Chamorro A. Anterior cerebral artery disease. Stroke 2004:101-22

12 Burlakoti A, Kumaratilake J, Taylor J, et al. The cerebral basal arterial network: morphometry of inflow and outflow components. J Anat 2017;230:833-41.

13 Okahara M, Kiyosue $\mathrm{H}$, Mori $\mathrm{H}$, et al. Anatomic variations of the cerebral arteries and their embryology: a pictorial review. Eur Radiol 2002;12:2548-61.
14 Anand D, Cordina SM. Intracranial aneurysms and their relationship to circle of Willis variations. Stroke 2015;46:AWP81.

15 van Laar PJ, Hendrikse J, Golay X, et al. In vivo flow Territory mapping of major brain feeding arteries. Neuroimage 2006;29:136-44.

16 Hendrikse J, van Raamt AF, van der Graaf Y, et al. Distribution of cerebral blood flow in the circle of Willis. Radiology 2005;235:184-9.

17 Sampath R, Vannemreddy P, Nanda A. Fusiform aneurysms of the anterior communicating artery: illustrative series of 5 cases with operative techniques. Neurosurgery 2010;67:ons407-15.

18 Dell S. Asymptomatic cerebral aneurysm: assessment of its risk of rupture. Neurosurgery 1982;10:162-6.

19 Gunnal SA, Farooqui MS, Wabale RN. Anatomical variations of the circulus arteriosus in cadaveric human brains. Neurol Res Int 2014;2014:1-16.

20 Brown RD, Broderick JP. Unruptured intracranial aneurysms: epidemiology, natural history, management options, and familial screening. The Lancet Neurology 2014;13:393-404.

21 Alnaes MS, Isaksen J, Mardal K-A, et al. Computation of hemodynamics in the circle of Willis. Stroke 2007;38:2500-5.

22 Krex D, Schackert H, Schackert G. Genesis of cerebral aneurysmsan update. Acta Neurochir 2001;143:429-49.

23 Schneider CA, Rasband WS, Eliceiri KW. NIH image to ImageJ: 25 years of image analysis: for the past 25 years $\mathrm{NIH}$ image and ImageJ software have been pioneers as open tools for the analysis of scientific images. we discuss the origins, challenges and solutions of these two programs, and how their history can serve to advise and inform other software projects. Nature Methods 2012;9:671-6.

24 Jamaiyah H, Geeta A, Safiza M, et al. Reliability and technical error of calf circumference and Mid-half arm span measurements for nutritional status assessment of elderly persons in Malaysia. Malaysian journal of nutrition 2008;14:137-50.

25 Jou L-D, Lee DH, Mawad ME. Cross-flow at the anterior communicating artery and its implication in cerebral aneurysm formation. J Biomech 2010;43:2189-95.

26 Brust JCM, Chamorro A. Anterior cerebral artery disease. Stroke 2011:362-83.

27 Kroon M, Holzapfel GA. A model for saccular cerebral aneurysm growth by collagen fibre remodelling. J Theor Biol 2007;247:775-87.

28 Yamaguchi R, Ujiie H, Haida S, et al. Velocity profile and wall shear stress of saccular aneurysms at the anterior communicating artery. Heart Vessels 2008;23:60-6.

29 Meng H, Wang Z, Hoi Y, et al. Complex hemodynamics at the apex of an arterial bifurcation induces vascular remodeling resembling cerebral aneurysm initiation. Stroke 2007;38:1924-31.

30 Imaizumi Y, Mizutani T, Shimizu K, et al. Detection rates and sites of unruptured intracranial aneurysms according to sex and age: an analysis of Mr angiography-based brain examinations of 4070 healthy Japanese adults. J Neurosurg 2018;34:1-6.

31 Payne KA, Huybrechts KF, Caro J, et al. Long term cost-of-illness in stroke. Pharmacoeconomics 2002;20:813-25.

32 Housepian EM, Poot JL. A systematic analysis OK intracranial aneurysms from the autopsy file of the Presbyterian Hospital 1914 to 1956. J Neuropathol Exp Neurol 1958;17:409-23.

33 Mascia L, Mazzeo AT, Caccia S. Critical care management of subarachnoid hemorrhage (SAH). practical trends in anesthesia and intensive care 2017: Springer 2018:147-69. 